\title{
Study on 3D reconstruction techniques of rocket body model based on strain monitor
}

\author{
Aihua Chen*, Long Cao, Guowei Yan and Ze Xu \\ Jiuquan Satellite Launch Center, Jiuquan 732750, Gansu, China
}

\begin{abstract}
A three-dimensional reconstruction technique based on strain monitoring is proposed in this paper. According to the strain data of finite measuring points, the shape characteristics of the whole rocket structure are reconstructed, which provides the basis for the reliability judgment of the structure. Based on the theory of $K o$, the $3 \mathrm{D}$ reconstruction algorithm for the rocket model of cylindrical structure is realized. The strain nephogram of plate model is simulated by ANASYS. And the strain nephogram is reconstructed by reconstruction algorithm, then compared with the simulation results. The results show that the maximum error between the results of reconstruction and simulation is $1.91 \%$, which indicated that the agreement is great.
\end{abstract}

\section{Introduction}

With the development of aerospace industry, the long-term storage, rapid unsealing and long-distance transportation of launch vehicles will become normal. In the process of longterm storage and long-distance transportation, the status change of rocket structure is an important impact to the rocket and it's execution of launch mission ${ }^{[1-2]}$. How to achieve the effective monitoring of the rocket structure can provide technical support for the rapid judgment of the reliability of the rocket structure.

At present, the feasible method is to use Fiber Bragg Grating Sensor to measure several strain data of the structure surface, and then obtain the change of structure shape based on method of modal superposition, inverse finite element and a three-dimensional(3D) reconstruction algorithm, so as to further realize the judgment of structural state. And the most important technology is how to effectively realize the $3 \mathrm{D}$ reconstruction of the structure. Some scholars have realized the dynamic analysis and 3D reconstruction ${ }^{[3-5]}$ of rockets, satellites and other spacecraft based on truss model, but the research on the whole model analysis and reconstruction of rocket structure is relatively rare. In this paper, through the research and improvement of the 3D reconstruction theory, the reconstruction of the cylindrical rocket structure plate model is effectively realized, and the results are in good agreement with the simulation analysis results.

\footnotetext{
* Corresponding author: aihuachen369@163.com
} 


\section{The theory of 3D reconstruction}

The dynamic response of rocket and the change of structural mechanical characteristic are mainly reflected by the change of configuration ${ }^{[6-7]}$.In order to realize the detection of rocket structure characteristics, it is necessary to solve the $3 \mathrm{D}$ reconstruction technology of its shape structure.

The structural deformation of cylindrical rocket model under external force belongs to small elastic deformation. It is difficult to reconstruct the 3D model directly by extracting the strain value on the surface of cylindrical structure. In order to solve this problem, we can extract a section of slender strip with width of $\Delta$ along the axis of the model, as shown in Fig.1, Because the $\Delta$ is small, it can be considered that the extracted local structure is a slender strip structure. By the idea of integral, the $3 \mathrm{D}$ reconstruction of the whole rocket model can be transformed into the reconstruction of several slender strip structures. the theory of $K o$ displacement can be used to achieve the deformation monitoring and shape reconstruction ${ }^{[8]}$. Through inversion of method, the rocket model can be reconstructed.

The rocker model of cylindrical structure

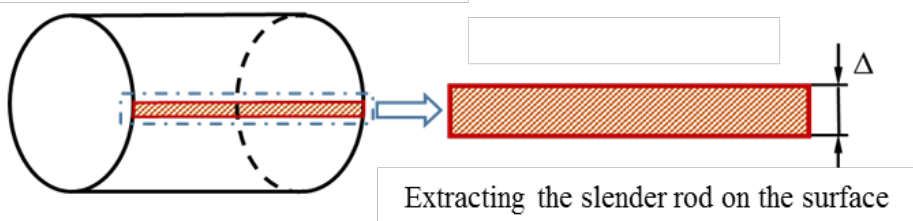

Fig. 1. Schematic diagram of partial plate structure extraction.

\subsection{Reconstruction algorithm based on Ko theory}

The reconstruction method which used Ko theory is through piecewise treatment to the whole structure. Firstly, the whole structure is divided into $n$ segments of deformation structure. By obtaining the strain data of each segment, the corresponding deflection information is calculated and recursively calculated. Finally, the overall deformation curve is obtained ${ }^{[9]}$.

According to the classical beam theory of material mechanics, under the condition of pure bending deformation or is the main deformation, it has the following relations:

$$
\frac{d^{2} y(x)}{d x^{2}}=M_{(x)} c / E I
$$

where $y$ is the longitudinal variation (deflection), $x$ is the transverse position, $M_{(x)}$ is the bending moment, $\varepsilon$ is the young's modulus and $I$ is the inertia. Where $c$ is the vertical distance from the structural surface to the structural neutral plane, which is defined as follows:

$$
c=\varepsilon_{t} h /\left(\varepsilon_{t}-\varepsilon_{b}\right)
$$

where $\varepsilon_{t}$ is the strain value measured on the upper surface of node $i$ of the structure, $\varepsilon_{b}$ is the strain value measured on the lower surface of node $i$ of the structure, and $h$ is the thickness of the structure. Generally, for the bending deformation of thin-walled structures, the strain values on the upper and lower surfaces are approximately equal, Therefore, the expression of $c$ can be simplified as follows: 


$$
c=h / 2
$$

And:

$$
\begin{gathered}
\varepsilon_{(x)}=\sigma_{(x)} / E=M_{(x)} c / E I \\
\sigma_{(x)}=M_{(x)} c / I
\end{gathered}
$$

The surface strain can be obtained by eliminating EI, the relationship between deflection and strain can be expressed as follows:

$$
\varepsilon_{(x)}=\mathrm{c} \frac{d^{2} y_{(x)}}{d x^{2}}
$$

Assuming that the measured strain values of starting point and end point on segment $i$ is $x_{i}^{\varepsilon}$ and $x_{i+1}^{\varepsilon}$. The strain distribution between the initial strain and the end strain can be obtained by linear interpolation

$$
\varepsilon_{(x)}=\varepsilon_{i}-\left(\varepsilon_{i}-\varepsilon_{i+1}\right) \cdot \frac{x-x_{i}^{\varepsilon}}{\Delta l_{i}}, \Delta l_{i}=x_{i+1}-x_{i}\left(x_{i}^{\varepsilon} \leq x \leq x_{i+1}^{\varepsilon}\right)
$$

The rotation angle $\theta_{i}^{\varepsilon}$ and deflection $y_{i}^{\varepsilon}$ of the starting point of the $i$ segment is given usually, In this case, the rotation angle $\theta_{(x)}$ can be obtained by one integration equation as follows:

$$
\tan \theta_{(x)}=\int_{x_{i}^{\varepsilon}}^{x} \frac{d^{2} y_{(x)}}{d x^{2}} d x+\tan \theta_{i}^{\varepsilon}=\int_{x_{i}^{\varepsilon}}^{x} c \varepsilon_{(x)} d x+\tan \theta_{i}^{\varepsilon},\left(x_{i}^{\varepsilon} \leq x \leq x_{i+1}^{\varepsilon}\right)
$$

To complete the integration of equation (8), we need to know the rotation angle of the starting point $\theta_{i}^{\varepsilon}$ and deflection $y_{i}^{\varepsilon}$. In order to complete the above calculation again, the piecewise fitting method is used. Under the bending condition of single side clamped plate structure, the boundary conditions at the starting point are as follows: $\theta_{1}^{\varepsilon}=0, y_{1}^{\varepsilon}=0$. Therefore, the calculation of the first deflection of the structure can be obtained by the following formula:

$$
y_{(x)}=\int_{x_{1}^{\varepsilon}}^{x} \tan \theta_{(x)} d x+y_{1}^{\varepsilon}=2 \int_{x_{1}^{\varepsilon}}^{x} \int_{1}^{\varepsilon} \frac{\varepsilon_{(x)}^{\varepsilon}}{h} d x d x+\int_{x_{1}^{\varepsilon}}^{x} \tan \theta_{1}^{\varepsilon} d x+y_{i}^{\varepsilon},\left(x_{1}^{\varepsilon} \leq x \leq x_{2}^{\varepsilon}\right)
$$

Because of $\theta_{1}^{\varepsilon}=0, \quad y_{1}^{\varepsilon}=0$, the above formula can be expressed as follows:

$$
y_{(x)}=\int_{x_{1}^{\varepsilon}}^{x} \int_{x_{1}^{\varepsilon}}^{x} c \varepsilon_{(x)} d x d x
$$


Based on equation (10), the deflection of any point on the first segment can be obtained, and then the above process can be repeated. Based on equation (9), the deflection of any point on segment $n$ can be obtained, and the strip curve can be reconstructed. The deformation surface of the whole structure can be reconstructed by reconstructing multi strip variable curves with equal interval and horizontal interpolation.

\subsection{ANASYS model simulation of plate structure model}

As shown in Fig.2, the infinitestimal of rocket structure are extracted along the axial direction, which can be regarded as plate-shaped structure. Assumed that the extracted part is $100 \mathrm{~mm}$ long, $5 \mathrm{~mm}$ wide and $1 \mathrm{~mm}$ thick. The material parameters are: The elastic modulus is $E=2.0 \times 10^{11} \mathrm{~Pa}$, The Poisson's ratio is 0.3 and density is $\rho_{0}=7850 \mathrm{~kg} / \mathrm{m}^{3}$. As shown in Fig.2, Fixed constraints are applied on the left end of the plate-shaped model. The force $\mathrm{F}$ downward along the $\mathrm{y}$-axis is applied to the right free end face, which amplitude is $10 \mathrm{~N}$ and frequency is $60 \mathrm{~Hz}$. The function of $\mathrm{F}$ changed in 1 second is as follows:

$$
F=10 \sin (120 \pi t), 0<t<1
$$

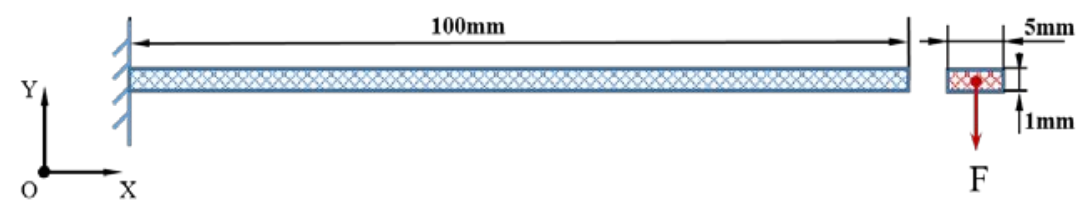

Fig. 2. Force diagram of plate structure.

The transient dynamic analysis of the state of the above model is carried out by using Anasys. The simulation results are shown in Fig. 3.
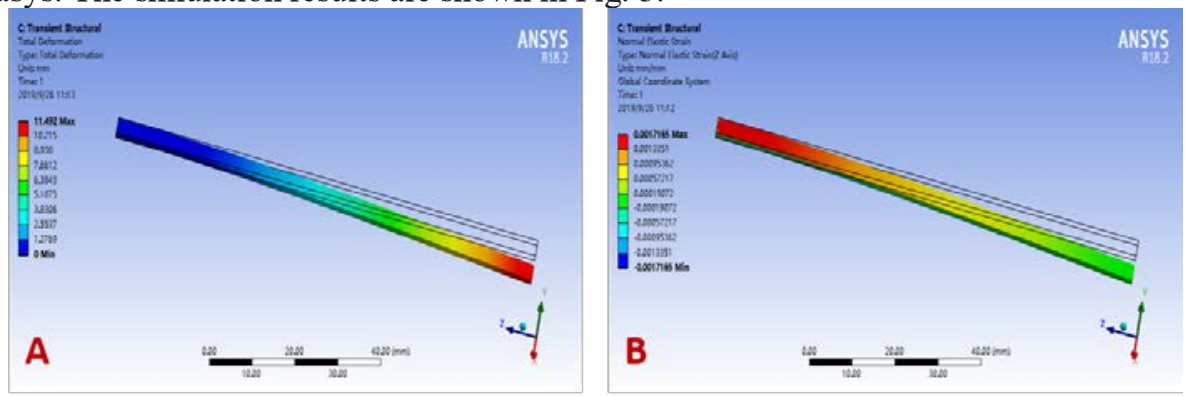

Fig. 3. Anasys simulation results.

In Fig.3, Figure A is the total deformation nephogram of the plate-shaped structure. It can be seen that when the left end face is fixed and the right endpoint is applied with vertical downward variable load, the maximum deformation value is at the free endpoint, and the maximum value is $11.492 \mathrm{~mm}$; Figure B is the normal strain nephogram, the maximum strain is at the fixed endpoint, and the maximum value is $0.00172 \mathrm{~mm} / \mathrm{mm}$.

\section{Verification of reconstruction algorithm}

As shown in Fig.4, In order to prove the 3D reconstruction effect of the algorithm, firstly, from the deformation and strain simulation results of the third section, data points are extracted from the upper surface of the plate structure along the central line and two sidelines at an equal interval of $4 \mathrm{~mm}$. Then the strain of each group of data is reconstructed 
according to the strain data, and compared with the strain curve obtained by simulation; finally, based on three strain curves, the plate structure is reconstructed by the method of longitudinal interpolation.

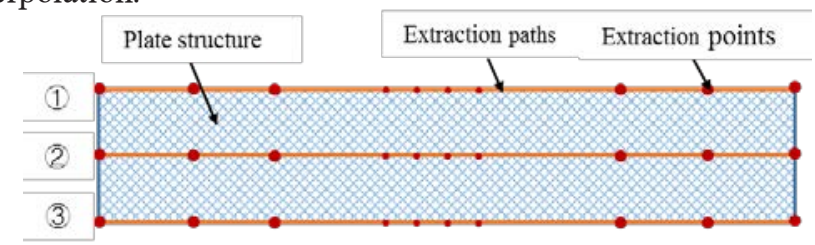

Fig. 4. Data point extraction method.

Fig.5 shows the comparison between the reconstruction and the simulation results of curves (1), (2) and (3). It can be seen that the reconstruction results of each curve are in good agreement with the simulation results.

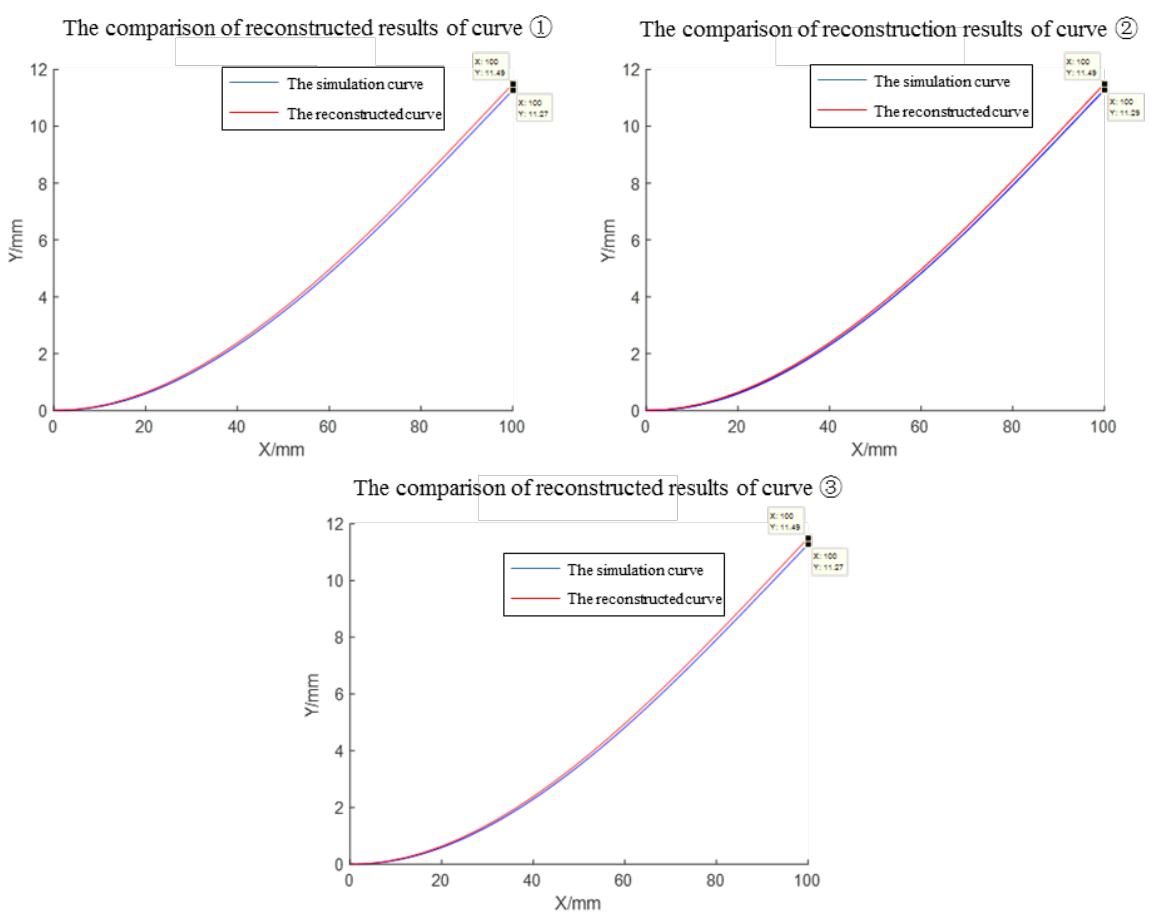

Fig. 5. Comparison of curve reconstruction results.

It can be seen from table 1 that the reconstruction result of curve (2) is the best with an error of $1.74 \%$. Because the model of plate structure is symmetrical, the reconstruction results of curve (1) and curve (3) is consistent, with the error of 1.91. The main reason which caused the small error between curve (2) and curve (1)/(3) is that the concentrated stress exists at the left fixed endpoint of the plate, which makes the strain value at the edge larger than the middle, which leads to different reconstruction results.

Table 1. Comparison results of reconstruction and simulation

\begin{tabular}{cccc}
\hline Curve number & Maximum reconstruction result & Maximum result simulation & error \\
\hline 1 & 11.27 & 11.49 & $1.91 \%$ \\
\hline$(2)$ & 11.29 & 11.49 & $1.74 \%$ \\
\hline 3 & 11.27 & 11.49 & $1.91 \%$ \\
\hline
\end{tabular}




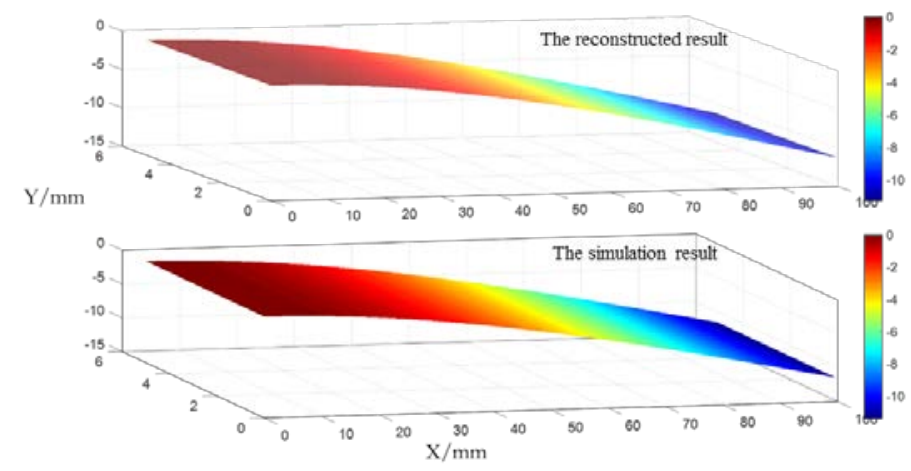

Fig. 6. Reconstruction results and simulation results.

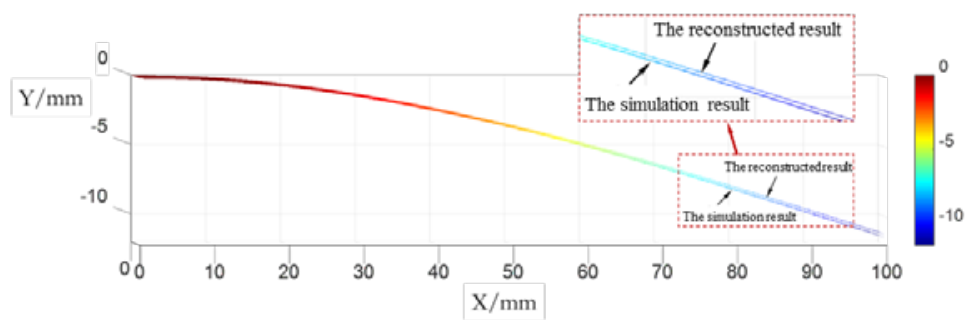

Fig. 7. Comparison between reconstruction results and simulation results.

Matlab is used to deal with the obtained data. Fig. 6 shows the reconstruction rand simulation results, and Fig.7 shows the comparison between the reconstruction structure and simulation results. It can be seen that the results of 3D reconstruction which using the extracted data is in good agreement with the simulation results, which demonstrates that the algorithm can achieve the $3 \mathrm{D}$ reconstruction based on strain data of plate effectively.

\section{Conclusion}

In this paper, Based on displacement deflection theory of Ko, the $3 \mathrm{D}$ reconstruction of local plate structure of cylindrical rocket model is realized. In order to verify the effectiveness of the method, the strain nephogram and total deformation nephogram of the plate model are obtained by using Anasys, and the finite data points are extracted, which is compiled by using matlab, compared with the results of simulation, the maximum error is $1.91 \%$, and the reconstruction coincidence is great.

\section{References}

1. Liu X.W., Zhang G.H. Construction of space emergency launch system. Naitonal defense science \& technology. 37,14 (2016)

2. He S.F, Gu Z.F, Li K.Z, etc. Integrated design of maneuver launch position and time for operationally responsive solid launch vehicle. Aerospace Control. 38, 61 (2020)

3. Pan Z.W., Wang X, Xing Y.F. A beam model based longitudinal-lateral-torsional inergrated modelling technique for launch. Journal of Astronautics. 31, 1310 (2010)

4. Zhang X, Wu Z.G, Yang C. Based on the equivalent Liang Jianmo for energy method and dynamic analysis. CARS. 5, 266 (2009) 
5. Noor A K, Nemeth M P. Analysis of spatial beamlike lattices with rigid joints. Computer Methods in Applied Mechanics and Engineering. 24, 35 (1980).

6. Chen Y, Xiong K, Wang X.W, etc. Progress and Challenges in Aeronautical Smart Structure Systems. ACTA AERONAUTICA ET ASTIONAUTICA SINICA. 25, 21 (2004)

7. Noor A K, Mikulas M M. Large Space Structures: Dynamics and Control. Springer Berlin Heidelberg. 1-34 (1988).

8. Li D.T. Flexible structure deformation monitoring technology research based on distributed optical fiber. Nanjing: Nanjing University of Aeronautics and Astronautics. (2017)

9. William L.Ko, W.L.Richards, Van T.Tran. Displacement theories for in-flight deformed shape predictions of a long span flying wing. Edwards, California: NASA Dryden Flight Research Center. (2007) 